\section{Welche Infektionsprophylaxe wählen Sie bei der transrektalen Prostatabiopsie?}

\author{
Antibiotika gelten als Mittel der Wahl, um vor allem Allgemeininfektionen in- \\ folge von Prostatabiopsien zu vermeiden. Urologen greifen dabei vorwiegend \\ auf Fluorchinolone zurück. Das könnte sich schon bald ändern.
}

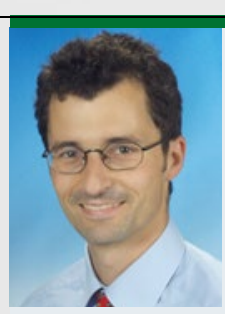

Diese Frage

beantwortet für Sie

Prof. Dr. med. Florian

Wagenlehner

Klinik und Poliklinik für Urologie, Kinderurologie, Andrologie, Universitätsklinikum Gießen und Marburg, Rudolf-Buchheim-Str. 7, 35392 Gießen E-Mail: Florian.Wagenlehner@ chiru.med.uni-giessen.de
D e Prostatabiopsie entwickelte sich im Laufe der Jahre zum Goldstandard, um Prostatakarzinome zu diagnostizieren. Weltweit beträgt die Zahl der jährlich durchgeführten Biopsien mittlerweile mehrere Millionen. Am häufigsten nutzen Urologen dabei den transrektalen Zugang. Um das Infektionsrisiko des operativen Verfahrens einzudämmen, erhalten Patienten prophylaktisch Antibiotika. In placebokontrollierten Studien aus den 1990er-Jahren wiesen Forscher nach, dass vor allem die präoperative antibiotische Prophylaxe mit Fluorchinolonen die Infektionsraten verringerte. Die Rate von schweren, fieberhaften Infektionen lag hierbei unter $1 \%$. Eine prolongierte Prophylaxe (mehrere Tage) zeigte keinen besseren Schutz vor Infektionen.

\section{Flurochinolone nur noch eingeschränkt verwendbar} In den letzten Jahren berichteten Wissenschaftler verschiedener retrospektiver und prospektiver Studien über ansteigende Infektionsraten nach transrektalen Prostatabiopsien. Übereinstimmend beobachteten sie fieberhafte Infektionen bei 3-5\%, die Mortalitätsrate lag bei circa $1 \%$. Der vorherrschende Risikofaktor für schwere Infektionen scheinen Flu-

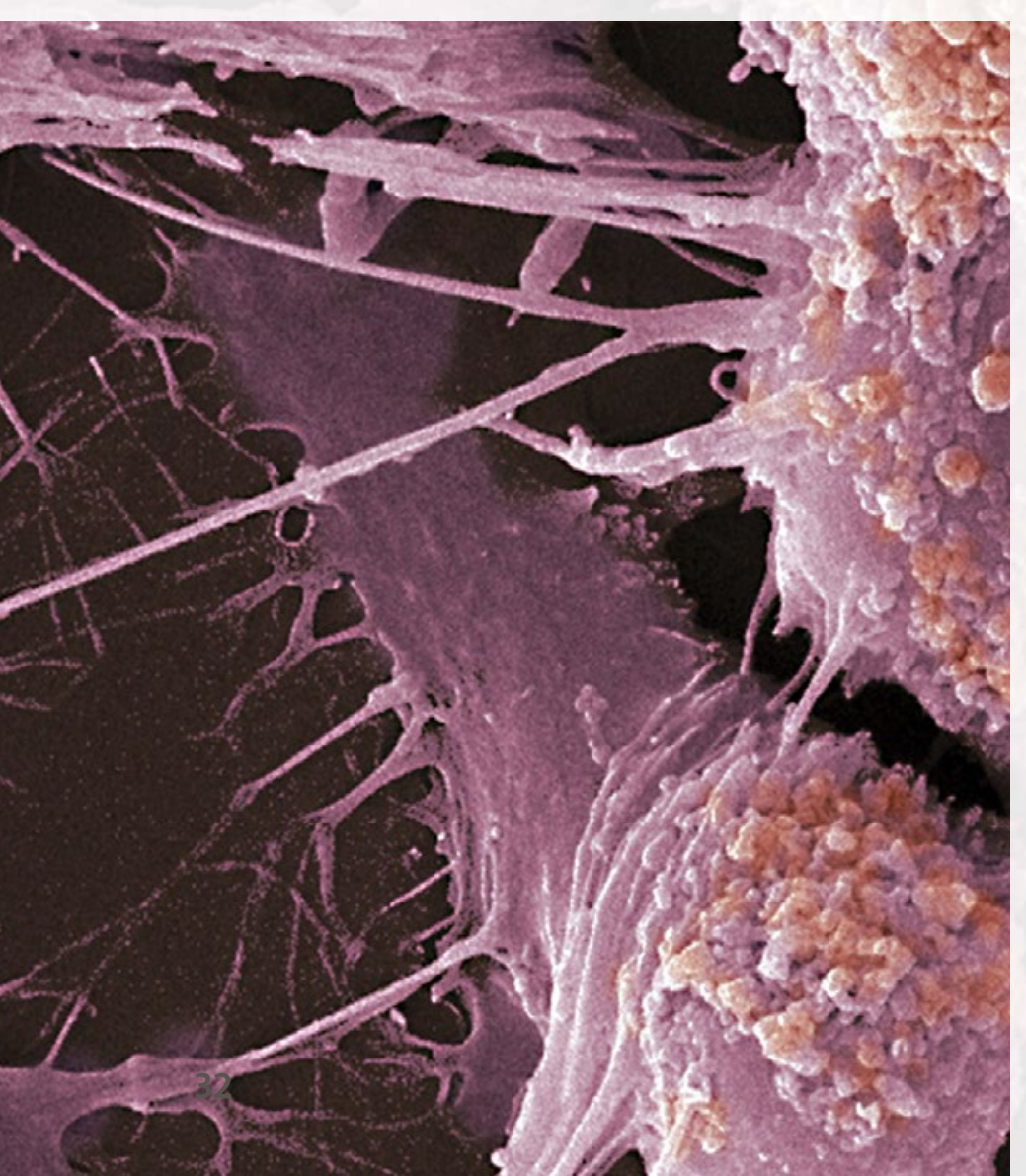

orchinolon-resistente Bakterien im Stuhl zu sein. Dies würde auch die Korrelation der zunehmenden Infektionsraten mit der ansteigenden Fluorchinolonresistenz über die letzten Jahre erklären. Deswegen sollten nur diejenigen Patienten vor der Prostatabiopsie prophylaktisch Flurochinolonen erhalten, bei denen nicht das Risiko besteht, dass Fluorchinolon-resistente Bakterien in der fäkalen Flora vorliegen. Ein gesichert erhöhtes Risiko für Fluorchinolon-resistente Bakterien haben Patienten, die im letzten Jahr Fluorchinolone, unabhängig der Indikation, erhalten haben sowie Patienten, die eine Reiseanamnese in Hochresistenzgebiete wie beispielsweise Asien oder Südeuropa aufweisen. Ob Fluorchinolon-resistente Bakterien in der fäkalen Flora vorhanden sind, kann aber auch mikrobiologisch getestet werden, indem die fäkale Flora mit Rektumabstrich auf Fluorchinolon-Selektivnährböden gescreent wird. Wachsen Bakterien auf diesen Selektivnährböden sind diese automatisch Flurochinolon-resistent. In diesen Fällen sollten keine Fluorchinolone prophylaktisch verabreicht werden. Aktuelle - jedoch begrenzte - Daten stützen diesen Ansatz.

\section{Alternativen auf dem Prüfstand}

Empfehlungen zu alternativen Antibiotika gestalten sich schwierig, da eine ausreichende Evidenz aus kontrollierten Studien praktisch nicht vorhanden ist. Deswegen bleibt derzeit nichts anderes übrig, als zielgerichtete Parameter für die alternative Antibiotikaauswahl zu diskutieren:

1. Da Enterobakterien die hauptsächlichen Erreger bei infektiösen Komplikationen darstellen, sollte das zu auszuwählende Antibiotikum gegen diese wirksam sein.

2.Verschiedene Daten legen nahe, dass das erforderliche Antibiotikum zudem eine Aktivität in der nicht entzündeten Prostata aufweisen sollte. Diese ist bei Fluorchinolonen am höchsten, eine gewisse Aktivität findet sich aber auch bei Trimethoprim/Sulfamethoxazol, Beta-Laktamantibiotika wie Breitspektrumpenicilline, Cephalosporine oder auch Fosfomycin. Diese Antibiotika könnten deshalb unter Umständen alternativ diskutiert werden.

Mit adjunktiven Verfahren, wie einer Enddarmreinigung, erreichte man bisher keine eindeutige signifikante Reduktion der Infektionsraten, auch wenn in einzelnen Studien eine gesenkte Infektionsrate beschrieben wurde.

Ein perinealer Ansatz der Prostatastanzbiopsie könnte eine Alternative aufzeigen. In größeren Kohortenstudien waren infektiöse Komplikationen sehr niedrig, vergleichende Studien (transrektal versus transperineal) waren bisher allerdings nicht auf den Endpunkt infektiöse Komplikationen ausgelegt. 